\title{
STABILITY OF THE DENJOY-WOLFF THEOREM
}

\author{
Argyrios Christodoulou and Ian Short \\ Queen Mary University of London, School of Mathematical Sciences \\ London, E1 4NS, United Kingdom; argyrios.christodoulou@qmul.ac.uk \\ The Open University, School of Mathematics and Statistics \\ Milton Keynes, MK7 6AA, United Kingdom; ian.short@open.ac.uk
}

\begin{abstract}
The Denjoy-Wolff theorem is a foundational result in complex dynamics, which describes the dynamical behaviour of the sequence of iterates of a holomorphic self-map $f$ of the unit disc D. Far less well understood are nonautonomous dynamical systems $F_{n}=f_{n} \circ f_{n-1} \circ \cdots \circ f_{1}$ and $G_{n}=g_{1} \circ g_{2} \circ \cdots \circ g_{n}$, for $n=1,2, \ldots$, where $f_{i}$ and $g_{j}$ are holomorphic self-maps of $\mathbf{D}$. Here we obtain a thorough understanding of such systems $\left(F_{n}\right)$ and $\left(G_{n}\right)$ under the assumptions that $f_{n} \rightarrow f$ and $g_{n} \rightarrow f$. We determine when the dynamics of $\left(F_{n}\right)$ and $\left(G_{n}\right)$ mirror that of $\left(f^{n}\right)$, as specified by the Denjoy-Wolff theorem, thereby providing insight into the stability of the Denjoy-Wolff theorem under perturbations of the map $f$.
\end{abstract}

\section{Introduction}

Fundamental to this paper is the Denjoy-Wolff theorem (see, for example, [8, Theorem 5.4]), which can be stated as follows.

Theorem A. Suppose that $f$ is a holomorphic self-map of the open unit disc $\mathbf{D}$. Then either

(1) $f$ is the identity function or an elliptic Möbius transformation that fixes $\mathbf{D}$, or

(2) there exists a point $\zeta \in \overline{\mathbf{D}}$ such that the sequence of iterates $f, f^{2}, f^{3}, \ldots$ converges locally uniformly on $\mathbf{D}$ to $\zeta$.

To explain the terminology in this theorem, an elliptic Möbius transformation that fixes $\mathbf{D}$ is a conformal automorphism of $\mathbf{D}$ that is conjugate by another conformal automorphism to a rotation about the origin. For each positive integer $n$, the $n$th iterate $f^{n}$ of a holomorphic map $f$ is the function obtained by composing $f$ with itself $n$ times, $f^{n}=f \circ f \circ \cdots \circ f$. The theorem states that the iterates $f, f^{2}, f^{3}, \ldots$ converge locally uniformly on $\mathbf{D}$ to $\zeta$, meaning that the sequence of functions $f, f^{2}, f^{3}, \ldots$ converges uniformly on compact subsets of $\mathbf{D}$ to $\zeta$, using the Euclidean metric on $\overline{\mathbf{D}}$. In case (2), the point $\zeta$ is called the Denjoy-Wolff point of $f$; if $\zeta \in \mathbf{D}$ then it is a fixed point of $f$.

Our objective is to examine the stability of Theorem A under perturbations of the holomorphic map $f$, in a sense to be made precise shortly. We denote by $\mathcal{H}(\mathbf{D}, \mathbf{C})$ the topological space of all holomorphic maps from $\mathbf{D}$ to the complex plane $\mathbf{C}$, equipped with the compact-open topology. In $\mathcal{H}(\mathbf{D}, \mathbf{C})$, a sequence $\left(f_{n}\right)$ converges to a map $f$ if and only if $f_{n} \rightarrow f$ locally uniformly on $\mathbf{D}$.

We focus on the subspace $\mathcal{H}(\mathbf{D})$ of holomorphic self-maps of $\mathbf{D}$. If $\left(f_{n}\right)$ is a sequence in $\mathcal{H}(\mathbf{D})$ that converges locally uniformly on $\mathbf{D}$ to a map $f$, then either

https://doi.org/10.5186/aasfm.2021.4621

2020 Mathematics Subject Classification: Primary 30D05; Secondary 30F45.

Key words: Denjoy-Wolff theorem, holomorphic map, hyperbolic metric. 
$f \in \mathcal{H}(\mathbf{D})$ or else $f$ is a constant function with value on the boundary of $\mathbf{D}$ (see [3, Lemma 2.1]).

Given sequences $\left(f_{n}\right)$ and $\left(g_{n}\right)$ in $\mathcal{H}(\mathbf{D})$, we define the left-composition sequence generated by $\left(f_{n}\right)$ and the right-composition sequence generated by $\left(g_{n}\right)$ to be the sequences

$$
F_{n}=f_{n} \circ f_{n-1} \circ \cdots \circ f_{1} \text { and } G_{n}=g_{1} \circ g_{2} \circ \cdots \circ g_{n}, \quad n=1,2, \ldots,
$$

respectively. Sequences of this type arise in a variety of contexts in dynamical systems, with differing notations and terminology. In future we omit the o symbol from compositions.

The dynamical behaviour of the sequence of iterates $\left(f^{n}\right)$, where $f \in \mathcal{H}(\mathbf{D})$, depends on whether $f$ is the identity function, an elliptic Möbius transformation, or if it has a Denjoy-Wolff point that lies in $\mathbf{D}$ or on the boundary of $\mathbf{D}$. We determine whether the dynamics of $\left(F_{n}\right)$ and $\left(G_{n}\right)$ are similar to that of $\left(f^{n}\right)$ under the assumptions that $f_{n} \rightarrow f$ and $g_{n} \rightarrow f$. We find that, in a sense, right-composition sequences are more stable than left-composition sequences when $f$ has a Denjoy-Wolff point inside $\mathbf{D}$, but the reverse holds when the Denjoy-Wolff point of $f$ lies on the boundary of $\mathbf{D}$. When $f$ is the identity function, there is similar stability for both left- and right-composition sequences.

We make significant use of the hyperbolic metric on $\mathbf{D}$, which is the Riemannian metric $2|d z| /\left(1-|z|^{2}\right)$. We denote the corresponding distance function by $\rho$. Crucial to our study is the Schwarz-Pick lemma, which says that if $f \in \mathcal{H}(\mathbf{D})$, then $\rho(f(z), f(w)) \leq \rho(z, w)$, for $z, w \in \mathbf{D}$, with equality if and only if $f$ is a conformal automorphism of $\mathbf{D}$. If $f$ is not a conformal automorphism, then for each compact subset $K$ of $\mathbf{D}$ we can find a positive constant $k<1$ such that $\rho(f(z), f(w)) \leq k \rho(z, w)$, for $z, w \in K$.

There is an extensive literature on stability results for holomorphic dynamical systems; we draw attention to the papers of Beardon [2], Gill [5, 6] and Pommerenke [9] for work closest to our own. Beardon and Gill were motivated in part by the theory of limit-periodic continued fractions, in which one considers the stability of continued fractions under perturbations of the coefficients. In [2], Beardon looks at the stability of Möbius transformations under iteration. We develop the geometric approach of [2], and apply it to the class of holomorphic maps, which is far larger and more complex than the class of Möbius transformations. Note that Theorem 3.1 of Section 3 could be deduced quickly from [2, Theorem 4.7] (the proof we give is short anyway).

Gill $[5,6]$ studies composition sequences of holomorphic maps for which the constituent maps approach a limit function. Using Euclidean estimates he obtains results of a similar type to Theorems 3.1 and 3.3. One of the benefits of our geometric approach is that we obtain strong results with succinct statements and concise proofs using the hyperbolic metric.

Pommerenke [9] considers right-composition sequences $\left(F_{n}\right)$ under the assumption that $f_{n} \rightarrow f$, for some non-elliptic map $f$, and attempts to find constants $a_{n}$ and $b_{n}$ such that $a_{n} F_{n}+b_{n} \rightarrow F$, for some non-constant function $F$. Whether this is possible depends on the nature of the Denjoy-Wolff point of $f$. Our objectives are somewhat tangential to this, such that we obtain a complete analysis of stability for both left- and right- composition sequences and any choice of holomorphic map $f$. 


\section{Stability at elliptic transformations and the identity function}

Here we consider the behaviour of the left- and right-composition sequences $F_{n}=$ $f_{n} f_{n-1} \cdots f_{1}$ and $G_{n}=g_{1} g_{2} \cdots g_{n}$, where $f_{n}, g_{n} \in \mathcal{H}(\mathbf{D})$, under the assumption that the sequences $\left(f_{n}\right)$ and $\left(g_{n}\right)$ converge to an elliptic Möbius transformation fixing $\mathbf{D}$ or the identity function $I$. We focus particularly on the latter case, because the iterates of an elliptic transformation do not themselves converge in $\mathcal{H}(\mathbf{D}, \mathbf{C})$.

The next example demonstrates that when $f_{n} \rightarrow I$, and without further assumptions, the sequence $\left(F_{n}\right)$ can behave erratically.

Example 2.1. Let $f_{n}(z)=e^{i / n} z$, for $n=1,2, \ldots$, so $f_{n} \rightarrow I$. Then

$$
F_{n}(z)=\lambda_{n} z, \quad \text { where } \quad \lambda_{n}=e^{i\left(1+\frac{1}{2}+\cdots+\frac{1}{n}\right)} .
$$

The sequence $\left(\lambda_{n}\right)$ forms a dense subset of the unit circle. Consequently, the sequence $\left(F_{n}\right)$ accumulates at the identity function and every rotation of the unit circle.

Essentially the same example can be used with $g_{n}$ in place of $f_{n}$ and $G_{n}$ in place of $F_{n}$, because the functions commute.

We can get quite different behaviour with other choices for functions $f_{n} \rightarrow I$. For example, choosing $f_{n}(z)=(1-1 / n) z$, for $n=2,3, \ldots$, we see that $\left(F_{n}\right)$ converges locally uniformly on $\mathbf{D}$ to 0 .

Example 2.1 indicates that to obtain more controlled behaviour of $\left(F_{n}\right)$ and $\left(G_{n}\right)$ under the assumption that $f_{n} \rightarrow I$ and $g_{n} \rightarrow I$ we need additional constraints on convergence. Theorems 2.2 and 2.3 , to follow, show that such control can be achieved if we stipulate that the convergence is sufficiently fast (in a sense to be made precise). In fact, using the following result from [4, Theorem 1.1], we will see that it is sufficient to assume that $\left(f_{n}\right)$ and $\left(g_{n}\right)$ converge to the identity function suitably fast at just two points in $\mathbf{D}$.

Theorem B. Suppose that $f, g \in \mathcal{H}(\mathbf{D})$, with $g$ a conformal automorphism of $\mathbf{D}$, and $a, b, z \in \mathbf{D}$, with $a \neq b$. Then

$$
\rho(f(z), g(z)) \leq \lambda(\rho(f(a), g(a))+\rho(f(b), g(b))),
$$

where

$$
\lambda=\frac{\exp (\rho(z, a)+\rho(a, b)+\rho(b, z))}{\rho(a, b)} .
$$

We now state our first result about stability of the Denjoy-Wolff theorem at the identity function or an elliptic transformation, for left-composition sequences.

Theorem 2.2. Suppose that $f$ is either the identity function or an elliptic Möbius transformation that fixes $\mathbf{D}$, and $f_{1}, f_{2}, \ldots$ are non-constant holomorphic self-maps of $\mathbf{D}$ for which

$$
\sum_{n=1}^{\infty} \rho\left(f_{n}(a), f(a)\right)<+\infty \text { and } \sum_{n=1}^{\infty} \rho\left(f_{n}(b), f(b)\right)<+\infty
$$

for two distinct points $a, b \in \mathbf{D}$. Then the sequence $\left(f^{-n} F_{n}\right)$, where $F_{n}=f_{n} f_{n-1} \cdots f_{1}$, converges locally uniformly on $\mathbf{D}$ to a non-constant holomorphic self-map of $\mathbf{D}$.

Proof. Let $d=\frac{1}{3} \rho(a, b)$ and let $K$ be a closed hyperbolic disc that is centred at a fixed point of $f$ and contains $a$ and $b$. Observe that if $z \in K$, then $f^{n}(z) \in K$, for $n \in \mathbf{Z}$. By applying Theorem B to the functions $f_{n}$ and $f$, for $n=1,2, \ldots$, we see 
that

$$
\sum_{n=1}^{\infty} \sup _{z \in K} \rho\left(f_{n}(z), f(z)\right)<+\infty
$$

Notice that it suffices to prove the theorem for the truncated left-composition sequence with $n$th term $f_{n} f_{n-1} \cdots f_{N}$, where $N$ is a fixed positive integer. In light of this observation, we may assume (after relabelling the functions) that in fact

$$
\sum_{n=1}^{\infty} \sup _{z \in K} \rho\left(f_{n}(z), f(z)\right)<d .
$$

Choose any point $z \in K$. Let $z_{n}=f^{n}(z)$, for $n=1,2, \ldots$ Then $z_{n} \in K$. Observe that

$$
\begin{aligned}
\rho\left(F_{n}(z), f^{n}(z)\right) & \leq \rho\left(f_{n} \cdots f_{1}(z), f_{n} \cdots f_{2} f(z)\right)+\rho\left(f_{n} \cdots f_{2}(f(z)), f^{n-1}(f(z))\right) \\
& \leq \rho\left(f_{1}(z), f(z)\right)+\rho\left(f_{n} \cdots f_{2}\left(z_{1}\right), f^{n-1}\left(z_{1}\right)\right),
\end{aligned}
$$

where, to obtain the second inequality, we have applied the Schwarz-Pick lemma with the function $f_{n} \cdots f_{2}$. Repeating this argument we see that

$$
\begin{aligned}
\rho\left(F_{n}(z), f^{n}(z)\right) \leq & \rho\left(f_{1}(z), f(z)\right)+\rho\left(f_{2}\left(z_{1}\right), f\left(z_{1}\right)\right)+\cdots \\
& +\rho\left(f_{n}\left(z_{n-1}\right), f\left(z_{n-1}\right)\right)<d
\end{aligned}
$$

for $n=1,2, \ldots$

Next, still with $z \in K$, we have

$$
\begin{aligned}
\rho\left(F_{n}(z), a\right) & \leq \rho\left(F_{n}(z), F_{n}(a)\right)+\rho\left(F_{n}(a), f^{n}(a)\right)+\rho\left(f^{n}(a), a\right) \\
& \leq \rho(z, a)+d+\rho\left(f^{n}(a), a\right) \leq l,
\end{aligned}
$$

for $n=1,2, \ldots$, where $l$ is three times the hyperbolic diameter of $K$. Similarly $\rho\left(F_{n}(z), b\right) \leq l$. Applying Theorem B to the functions $f_{n}$ and $f$, and with $F_{n-1}(z)$ in place of $z$, we obtain

$$
\rho\left(F_{n}(z), f\left(F_{n-1}(z)\right)\right) \leq \lambda\left(\rho\left(f_{n}(a), f(a)\right)+\rho\left(f_{n}(b), f(b)\right)\right),
$$

where

$$
\lambda=\frac{\exp \left(\rho\left(F_{n-1}(z), a\right)+\rho(a, b)+\rho\left(b, F_{n-1}(z)\right)\right)}{\rho(a, b)} \leq \frac{\exp (3 l)}{\rho(a, b)} .
$$

Consequently, we see that

$$
\sum_{n=1}^{\infty} \rho\left(f^{-n} F_{n}(z), f^{-(n-1)} F_{n-1}(z)\right)=\sum_{n=1}^{\infty} \rho\left(F_{n}(z), f\left(F_{n-1}(z)\right)\right)<\frac{2 d \exp (3 l)}{\rho(a, b)},
$$

for $z \in K$ (where $F_{0}$ is the identity function). Thus $\left(f^{-n} F_{n}\right)$ is a uniformly Cauchy sequence on $K$. Now, $K$ is an arbitrarily large compact subset of $\mathbf{D}$, so it follows that $\left(f^{-n} F_{n}\right)$ converges locally uniformly on $\mathbf{D}$ to a function $F$.

The function $F$ belongs to $\mathcal{H}(\mathbf{D})$, and it is not a constant function because $\rho\left(f^{-n} F_{n}(a), f^{-n} F_{n}(b)\right) \geq \rho(a, b)-\rho\left(f^{-n} F_{n}(a), a\right)-\rho\left(f^{-n} F_{n}(b), b\right)>3 d-d-d=d$, for $n=1,2, \ldots$, where we have applied inequality (2.1) to give $\rho\left(f^{-n} F_{n}(a), a\right)<d$ and $\rho\left(f^{-n} F_{n}(b), b\right)<d$.

When $f$ is the identity function $I$, Theorem 2.2 says that if $\sum \rho\left(f_{n}(a), a\right)<+\infty$ and $\sum \rho\left(f_{n}(b), b\right)<+\infty$, then the left-composition sequence $F_{n}=f_{n} f_{n-1} \cdots f_{1}$ converges locally uniformly on $\mathbf{D}$ to a non-constant holomorphic map $F \in \mathcal{H}(\mathbf{D})$. When $f$ is an elliptic transformation of finite order $m$, the theorem tells us that the sequence $\left(F_{n}\right)$ can be split into $m$ subsequences that converge to $F, f F, \ldots, f^{m-1} F$, 
respectively. For the remaining case, when $f$ is an elliptic transformation of infinite order, we see from Theorem 2.2 that $\left(F_{n}\right)$ accumulates at uncountably many different non-constant maps in $\mathcal{H}(\mathbf{D})$.

Next we state a result similar to Theorem 2.2 for right-composition sequences.

Theorem 2.3. Suppose that $g$ is either the identity function or an elliptic Möbius transformation that fixes $\mathbf{D}$, and $g_{1}, g_{2}, \ldots$ are non-constant holomorphic self-maps of $\mathbf{D}$ for which

$$
\sum_{n=1}^{\infty} \rho\left(g_{n}(a), g(a)\right)<+\infty \text { and } \sum_{n=1}^{\infty} \rho\left(g_{n}(b), g(b)\right)<+\infty
$$

for two distinct points $a, b \in \mathbf{D}$. Then the sequence $\left(G_{n} g^{-n}\right)$, where $G_{n}=g_{1} g_{2} \cdots g_{n}$, converges locally uniformly on $\mathbf{D}$ to a non-constant holomorphic self-map of $\mathbf{D}$.

Proof. Let $d=\frac{1}{3} \rho(a, b)$ and let $K$ be a closed hyperbolic disc that is centred at a fixed point of $g$ and that contains $a$ and $b$. By truncating the right-composition sequence $\left(G_{n}\right)$ by a fixed finite number of terms from the left (and relabelling the remaining functions), we can assume that

$$
\sum_{n=1}^{\infty} \sup _{z \in K} \rho\left(g_{n}(z), g(z)\right)<d .
$$

Now choose a point $z$ in $K$, and let $n$ be a positive integer. By applying the SchwarzPick lemma with the function $G_{n-1}$, we see that

$$
\rho\left(G_{n} g^{-n}(z), G_{n-1} g^{-(n-1)}(z)\right) \leq \rho\left(g_{n}(w), g(w)\right),
$$

where $w=g^{-n}(z)$ (and $G_{0}$ is the identity function). Since $w \in K$, it follows that

$$
\sum_{n=1}^{\infty} \rho\left(G_{n} g^{-n}(z), G_{n-1} g^{-(n-1)}(z)\right)<d .
$$

Therefore $\left(G_{n} g^{-n}\right)$ is a uniformly Cauchy sequence on $K$, and since $K$ can be chosen to be arbitrarily large, we deduce that $\left(G_{n} g^{-n}\right)$ converges locally uniformly on $\mathbf{D}$ to a function $G$.

This function $G$ belongs to $\mathcal{H}(\mathbf{D})$; we must show that it is not a constant function. To this end, we write $a_{n}=g^{-n}(a)$, for $n=1,2, \ldots$, and observe that

$$
\begin{aligned}
\rho\left(G_{n} g^{-n}(a), a\right) \leq & \rho\left(G_{n}\left(a_{n}\right), G_{n-1}\left(a_{n-1}\right)\right)+\rho\left(G_{n-1}\left(a_{n-1}\right), G_{n-2}\left(a_{n-2}\right)\right)+\cdots \\
& +\rho\left(G_{1}\left(a_{1}\right), a\right) \\
\leq & \rho\left(g_{n}\left(a_{n}\right), g\left(a_{n}\right)\right)+\rho\left(g_{n-1}\left(a_{n-1}\right), g\left(a_{n-1}\right)\right)+\cdots+\rho\left(g_{1}\left(a_{1}\right), g\left(a_{1}\right)\right),
\end{aligned}
$$

for $n=1,2, \ldots$, where, to obtain the second inequality, we applied the SchwarzPick lemma with the functions $G_{n-1}, G_{n-2}, \ldots G_{0}$, in that order. Since $a_{n} \in K$, for each index $n$, we find that $\rho\left(G_{n} g^{-n}(a), a\right)<d$, and similarly $\rho\left(G_{n} g^{-n}(b), b\right)<d$. Consequently,

$\rho\left(G_{n} g^{-n}(a), G_{n} g^{-n}(b)\right) \geq \rho(a, b)-\rho\left(G_{n} g^{-n}(a), a\right)-\rho\left(G_{n} g^{-n}(b), b\right)>3 d-d-d=d$, for $n=1,2, \ldots$ Hence $G$ is a non-constant holomorphic self-map of $\mathbf{D}$.

The special cases of Theorem 2.3 when the limit function $g$ is of finite order resemble the similar special cases of Theorem 2.2. In particular, when $g$ is the identity function, Theorem 2.3 says that if $\sum \rho\left(g_{n}(a), a\right)<+\infty$ and $\sum \rho\left(g_{n}(b), b\right)<+\infty$, then the right-composition sequence $G_{n}=g_{1} g_{2} \cdots g_{n}$ converges locally uniformly on $\mathbf{D}$ to a non-constant holomorphic self-map of $\mathbf{D}$. 


\section{Denjoy-Wolff point inside the disc}

In this section we consider the stability of the Denjoy-Wolff theorem at holomorphic functions that have a Denjoy-Wolff point inside the unit disc. Central to our approach is the following theorem from [1, Corollary 2.3] and [7, Theorem 1.2].

Theorem C. Suppose that $K$ is a compact subset of a simply connected hyperbolic domain $D$, and that $g_{1}, g_{2}, \ldots$ are holomorphic maps of $D$ into $K$. Then the right-composition sequence $G_{n}=g_{1} g_{2} \cdots g_{n}$ converges locally uniformly on $D$ to a constant in $K$.

Using Theorem $\mathrm{C}$ we obtain the following strong stability result for right-composition sequences.

Theorem 3.1. Let $g$ be a holomorphic self-map of $\mathbf{D}$ with a Denjoy-Wolff point $\zeta$ in $\mathbf{D}$. Then there is a neighbourhood $\mathcal{U}$ of $g$ in $\mathcal{H}(\mathbf{D})$ such that if $g_{1}, g_{2}, \ldots$ belong to $\mathcal{U}$, then the right-composition sequence $G_{n}=g_{1} g_{2} \cdots g_{n}$ converges locally uniformly on $\mathbf{D}$ to a constant in $\mathbf{D}$.

We use the notation $D(c, r)$ for the hyperbolic open disc with centre $c$ and hyperbolic radius $r$.

Proof. Let $D=D(\zeta, 1)$. Since $\bar{D}$ is a compact set in $\mathbf{D}$, we see from the Schwarz-Pick lemma that there is a positive constant $k<1$ (that depends on $\bar{D}$ ) with $\rho(g(z), g(w)) \leq k \rho(z, w)$, for $z, w \in \bar{D}$. Observe that $g$ fixes $\zeta$, so $g(D) \subset D(\zeta, k)$. Now choose a real number $s$ with $k<s<1$. Let

$$
\mathcal{U}=\{h \in \mathcal{H}(\mathbf{D}): h(D) \subset D(\zeta, s)\},
$$

a neighbourhood of $g$ in $\mathcal{H}(\mathbf{D})$, and let $K=\overline{D(\zeta, s)}$. If $g_{1}, g_{2}, \ldots$ belong to $\mathcal{U}$, then $g_{n}(D) \subset K$, for each index $n$, so we can apply Theorem $\mathrm{C}$ to see that the right-composition sequence $G_{n}=g_{1} g_{2} \cdots g_{n}$ converges locally uniformly on $D$ to a constant $\xi$ in $K$.

To prove that $\left(G_{n}\right)$ converges locally uniformly on $\mathbf{D}$ to $\xi$, we could now apply the Vitali-Porter theorem [10, Section 2.4]; however, it is easy enough to prove this assertion directly, as follows.

Suppose, in order to reach a contradiction, that $\left(G_{n}\right)$ does not converge locally uniformly on $\mathbf{D}$ to $\xi$. Then we can find a compact subset $L$ of $\mathbf{D}$, a positive number $\delta$, and a subsequence $\left(G_{n_{i}}\right)$, where $n_{1}<n_{2}<\cdots$, for which $G_{n_{i}}(L)$ is not contained in $D(\xi, \delta)$, for $i=1,2, \ldots$ However, $\left(G_{n}\right)$ is a normal family, so there is a further subsequence of $\left(G_{n_{i}}\right)$ that converges locally uniformly on $\mathbf{D}$ to some analytic function $G \in \mathcal{H}(\mathbf{D}, \mathbf{C})$. We know that $G(z)=\xi$, for $z \in D$, so in fact $G$ must be the constant function with value $\xi$. This contradicts the statement that $G_{n_{i}}(L)$ is not contained in $D(\xi, \delta)$, for $i=1,2, \ldots$, so we see that, contrary to our earlier assumption, $\left(G_{n}\right)$ does converge locally uniformally on $\mathbf{D}$ to $\xi$, as required.

The hypotheses of Theorem 3.1 can of course be weakened to assume that all but finitely many of the maps $g_{n}$ belong to $\mathcal{U}$.

The next example shows that there is no analogue of Theorem 3.1 for leftcomposition sequences.

Example 3.2. Consider the map $f(z)=z / 2$ with fixed point 0 . Let $\mathcal{U}$ be a neighbourhood of $f$ in $\mathcal{H}(\mathbf{D})$. We can choose a positive constant $\delta$ to be sufficiently small that any function $g(z)=z / 2+\mu$, where $\mu$ is a complex number with $|\mu| \leq \delta$, belongs to $\mathcal{U}$. 
Let $z_{n}=\delta e^{n i}$, for $n=1,2, \ldots$ The sequence $\left(z_{n}\right)$ is dense on the circle centred at the origin of radius $\delta$. Let $\delta_{1}=z_{1}$ and, for $n>1$, let $\delta_{n}=z_{n}-\frac{1}{2} z_{n-1}$, in which case

$$
\left|\delta_{n}\right|=\left|z_{n}-\frac{1}{2} z_{n-1}\right|=\delta\left|e^{i}-\frac{1}{2}\right|<\delta .
$$

It follows that the maps $f_{n}(z)=z / 2+\delta_{n}$ belong to $\mathcal{U}$.

Next, we prove by induction that the left-composition sequence $F_{n}=f_{n} f_{n-1} \cdots f_{1}$ satisfies $F_{n}(0)=z_{n}$, for $n=1,2, \ldots$ To see this, for $n=1$ we have $F_{1}(0)=\delta_{1}=z_{1}$, and for $n>1$, if $F_{n-1}(0)=z_{n-1}$, then

$$
F_{n}(0)=\frac{1}{2} F_{n-1}(0)+\delta_{n}=\frac{1}{2} z_{n-1}+\delta_{n}=z_{n} .
$$

Hence $\left(F_{n}(0)\right)$ diverges, and since $F_{n}(z)=F_{n}(0)+z / 2^{n}$, it follows that $\left(F_{n}\right)$ diverges pointwise on $\mathbf{D}$.

With slightly stronger hypotheses, however, we do obtain controlled behaviour of the left-composition sequence $\left(F_{n}\right)$.

Theorem 3.3. Let $f$ be a holomorphic self-map of $\mathbf{D}$ with a Denjoy-Wolff point $\zeta$ in $\mathbf{D}$. Suppose that $f_{1}, f_{2}, \ldots$ is a sequence of functions in $\mathcal{H}(\mathbf{D})$ that converges locally uniformly on $\mathbf{D}$ to $f$. Then the left-composition sequence $F_{n}=f_{n} f_{n-1} \cdots f_{1}$ converges locally uniformly on $\mathbf{D}$ to $\zeta$.

Proof. Let $K$ be a closed hyperbolic disc centred at $\zeta$. Observe that $f$ maps $K$ inside a smaller closed hyperbolic disc centred at $\zeta$. Since $f_{n} \rightarrow f$ uniformly on $K$ we see that $f_{n}$ maps $K$ inside itself for sufficiently large $n$. By truncating $F_{n}$ by finitely many terms on the right (and relabelling) we can assume that in fact $f_{n}(K) \subset K$ for all $n=1,2, \ldots$.

Since $K$ is compact, we see from the Schwarz-Pick lemma that there is a positive constant $k<1$ with $\rho(f(z), f(w)) \leq k \rho(z, w)$, for $z, w \in K$.

Choose $z \in K$. Observe that $f^{n}(z) \in K$ and $F_{n}(z) \in K$, for $n=1,2, \ldots$ Then

$$
\begin{aligned}
\rho\left(F_{n}(z), f^{n}(z)\right) & \leq \rho\left(F_{n}(z), f\left(F_{n-1}(z)\right)\right)+\rho\left(f\left(F_{n-1}(z)\right), f^{n}(z)\right) \\
& \leq \sup _{w \in K} \rho\left(f_{n}(w), f(w)\right)+k \rho\left(F_{n-1}(z), f^{n-1}(z)\right),
\end{aligned}
$$

for $n=1,2, \ldots$ Repeating this argument, we see that

$$
\begin{aligned}
\rho\left(F_{n}(z), f^{n}(z)\right) & \leq\left(1+k+k^{2}+\cdots+k^{n-1}\right) \sup _{w \in K} \rho\left(f_{n}(w), f(w)\right) \\
& \leq \frac{1}{1-k} \sup _{w \in K} \rho\left(f_{n}(w), f(w)\right)
\end{aligned}
$$

for $n=1,2, \ldots$ Since $\left(f_{n}\right)$ converges locally uniformly on $\mathbf{D}$ to $f$ we see that $\rho\left(F_{n}(z), f^{n}(z)\right) \rightarrow 0$ uniformly on $K$, so $F_{n} \rightarrow \zeta$ uniformly on $K$. Hence $\left(F_{n}\right)$ converges locally uniformly on $\mathbf{D}$ to the constant $\zeta$.

Notice that the left-composition sequence $\left(F_{n}\right)$ of Theorem 3.3 converges locally uniformly on $\mathbf{D}$ to $\zeta$, but the right-composition sequence $\left(G_{n}\right)$ of Theorem 3.1 converges to a constant that need not be $\zeta$. After all, adjusting $g_{1}$ causes the constant to change.

\section{Denjoy-Wolff point on the boundary of the disc}

This final section considers the stability of the Denjoy-Wolff theorem at holomorphic maps that have a Denjoy-Wolff point on the boundary of the unit disc. In a sense, this circumstance is the least stable of those considered so far, because one 
can find a holomorphic map $f$ with Denjoy-Wolff point on the boundary of $\mathbf{D}$, and a sequence of holomorphic maps $f_{1}, f_{2}, \ldots$ with $f_{n} \rightarrow f$, for which the behaviour of the left-composition sequence $F_{n}=f_{n} f_{n-1} \cdots f_{1}$ is erratic.

An example of this type follows, in which all the maps $f_{n}$ and $f$ are Möbius transformations. In this example we use the upper half-plane $\mathbf{H}$ in place of the unit disc $\mathbf{D}$, and work with the space of holomorphic self-maps of $\mathbf{H}$. We could transfer our example back to the unit disc by conjugating the maps $f_{n}$ and $f$ by the transformation $\phi(z)=(z-i) /(z+i)$, which is a conformal map from $\mathbf{H}$ to $\mathbf{D}$.

Example 4.1. Let $f(z)=z-1$, a holomorphic self-map of $\mathbf{H}$. It is a parabolic Möbius transformation with fixed point $\infty$, which is the Denjoy-Wolff point of $f$ (lying on the boundary of $\mathbf{H}$ in the extended complex plane $\mathbf{C}_{\infty}$ ). Let

$$
k_{n}(z)=\frac{n z-1}{z+n} \quad \text { and } \quad h_{n}=k_{n} f k_{n}^{-1},
$$

for $n=1,2, \ldots$ Then $h_{n}$ is also a parabolic Möbius transformation, with fixed point $n$. The maps $h_{n}$ are real Möbius transformations that fix $\mathbf{H}$, so they are conformal automorphisms of $\mathbf{H}$. Of course, they also act on $\mathbf{C}_{\infty}$ in the usual way.

Observe that $k_{n} \rightarrow I$, the identity transformation, so $h_{n} \rightarrow f$.

For $n=1,2, \ldots$, let $U_{n}=\{z:|z|<1 / n\}$ and $V_{n}=\{z:|z-n|<1 / n\}$. Choose a positive integer $r_{1}$ for which $h_{1}^{r_{1}}(i) \in V_{1}$. For each $n>1$, choose a positive integer $r_{n}$ such that $h_{n}^{r_{n}}\left(U_{n-1}\right) \subset V_{n}$. We can make these choices because $V_{n}$ is an open neighbourhood of the fixed point $n$ of $h_{n}$.

We define a sequence of Möbius transformations $f_{1}, f_{2}, \ldots$ as follows. The first $r_{1}$ maps in this sequence equal $h_{1}$ and the next 1 map equals $f$. Then the following $r_{2}$ maps in the sequence equal $h_{2}$ and the next 2 maps equal $f$. The following $r_{3}$ maps equal $h_{3}$ and the next 3 maps equal $f$, and so forth. Clearly $f_{n} \rightarrow f$ as $n \rightarrow \infty$. We will prove that the left-composition sequence $F_{n}=f_{n} f_{n-1} \cdots f_{1}$ diverges at $i$.

Since $h_{1}^{r_{1}}(i) \in V_{1}, f^{n}\left(V_{n}\right)=U_{n}$ and, for $n>1, h_{n}^{r_{n}}\left(U_{n-1}\right) \subset V_{n}$, it follows that

$$
h_{n}^{r_{n}} f^{n-1} h_{n-1}^{r_{n-1}} \cdots f^{1} h_{1}^{r_{1}}(i) \in V_{n}, \quad \text { whereas } \quad f^{n} h_{n}^{r_{n}} f^{n-1} h_{n-1}^{r_{n-1}} \cdots f^{1} h_{1}^{r_{1}}(i) \in U_{n},
$$

for $n=1,2, \ldots$. Consequently, there is a subsequence of $\left(F_{n}(i)\right)$ that converges to $\infty$ and there is another subsequence of $\left(F_{n}(i)\right)$ that converges to 0 . Hence $\left(F_{n}(i)\right)$ diverges.

Using the property that the maps $F_{n}$ preserve hyperbolic distance on $\mathbf{H}$, it can be shown that in fact $\left(F_{n}\right)$ diverges pointwise on $\mathbf{H}$; we omit the details.

Despite Example 4.1, the following theorem shows that, for a holomorphic map $f$ with Denjoy-Wolff point on the boundary of $\mathbf{D}$, if the convergence of $\left(f_{n}\right)$ to $f$ is sufficiently rapid, then the sequences $\left(F_{n}\right)$ and $\left(f^{n}\right)$ have similar dynamics.

Theorem 4.2. Let $f$ be a holomorphic self-map of $\mathbf{D}$ with a Denjoy-Wolff point $\zeta$ on the boundary of $\mathbf{D}$. Then there exist neighbourhoods $\mathcal{U}_{1}, \mathcal{U}_{2}, \ldots$ of $f$ in $\mathcal{H}(\mathbf{D})$ such that if $f_{n} \in \mathcal{U}_{n}$, for $n=1,2, \ldots$, then the left-composition sequence $F_{n}=$ $f_{n} f_{n-1} \cdots f_{1}$ converges locally uniformly on $\mathbf{D}$ to $\zeta$.

Proof. For each positive integer $n$, we define $D_{n}$ to be the open hyperbolic disc centred at 0 of radius $1+\rho\left(f^{n-1}(0), 0\right)$, and let

$$
\mathcal{U}_{n}=\left\{h \in \mathcal{H}(\mathbf{D}): \rho(h(z), f(z))<1 / 2^{n} \text { for } z \in D_{n}\right\}
$$

a neighbourhood of $f$ in $\mathcal{H}(\mathbf{D})$. Suppose that $f_{n} \in \mathcal{U}_{n}$, for $n=1,2, \ldots$ 
We will prove by induction on $m$ that

$$
\rho\left(F_{m}(0), f^{m}(0)\right)<1-\frac{1}{2^{m}}
$$

for $m=1,2, \ldots$ This is certainly true for $m=1$, by definition of $\mathcal{U}_{1}$. Suppose that it is true for the integer $m=n-1$, where $n>1$. Then

$$
\begin{aligned}
\rho\left(F_{n}(0), f^{n}(0)\right) & \leq \rho\left(F_{n}(0), f\left(F_{n-1}(0)\right)\right)+\rho\left(f\left(F_{n-1}(0)\right), f^{n}(0)\right) \\
& \leq \rho\left(F_{n}(0), f\left(F_{n-1}(0)\right)\right)+\rho\left(F_{n-1}(0), f^{n-1}(0)\right) \\
& <\rho\left(F_{n}(0), f\left(F_{n-1}(0)\right)\right)+1-\frac{1}{2^{n-1}},
\end{aligned}
$$

where we have applied the triangle inequality, the Schwarz-Pick lemma, and the induction hypothesis. Now, since

$$
\rho\left(F_{n-1}(0), 0\right) \leq \rho\left(F_{n-1}(0), f^{n-1}(0)\right)+\rho\left(f^{n-1}(0), 0\right)<1+\rho\left(f^{n-1}(0), 0\right),
$$

we see that $F_{n-1}(0) \in D_{n}$. So, by definition of $\mathcal{U}_{n}$, we have

$$
\rho\left(F_{n}(0), f\left(F_{n-1}(0)\right)\right)=\rho\left(f_{n}\left(F_{n-1}(0)\right), f\left(F_{n-1}(0)\right)\right)<\frac{1}{2^{n}} .
$$

Combining the inequalities obtained we conclude that

$$
\rho\left(F_{n}(0), f^{n}(0)\right)<\rho\left(F_{n}(0), f\left(F_{n-1}(0)\right)\right)+1-\frac{1}{2^{n-1}}<\frac{1}{2^{n}}+1-\frac{1}{2^{n-1}}=1-\frac{1}{2^{n}} .
$$

This completes the proof by induction.

A consequence of this observation is that $\rho\left(F_{n}(0), f^{n}(0)\right)<1$, for each positive integer $n$. Then, since $f^{n}(0) \rightarrow \zeta$, a point on the boundary of $\mathbf{D}$, we can use a formula for the hyperbolic metric in $\mathbf{D}$ such as

$$
\sinh \frac{1}{2} \rho(z, w)=\frac{|z-w|}{\sqrt{\left(1-|z|^{2}\right)\left(1-|w|^{2}\right)}},
$$

to see that $F_{n}(0) \rightarrow \zeta$ also.

Furthermore, we have that $\rho\left(F_{n}(z), F_{n}(0)\right) \leq \rho(z, 0)$, for any point $z \in \mathbf{D}$, and from this inequality we see that $\left(F_{n}\right)$ converges locally uniformly on $\mathbf{D}$ to $\zeta$ (with convergence in the Euclidean metric).

There is no such result as Theorem 4.2 for right-composition sequences. To see this, we provide an example that again uses the upper half-plane $\mathbf{H}$ in place of the unit disc D. We can make this switch because Theorem 4.2, like the other theorems, is conformally invariant, in the sense that one can obtain an equivalent theorem in $\mathbf{H}$ (or any other hyperbolic simply connected domain) by conjugating by a suitable conformal map.

Returning to the promised example, consider the function $g(z)=z+1$ acting on the upper half-plane $\mathbf{H}$ with Denjoy-Wolff point $\infty$. Let $h(z)=i+e^{2 \pi i z}$, which is a holomorphic self-map of $\mathbf{H}$ that satisfies $h g=h$. Now consider the right-composition sequence $G_{n}=g_{1} g_{2} \cdots g_{n}$, where $g_{1}=h$ and $g_{n}=g$, for $n>1$. Then $\left(g_{n}\right)$ converges to $g$ in the fastest possible way, but $G_{n}=h g^{n-1}=h$.

The following, similar example exhibits even worse behaviour of the sequence $\left(G_{n}\right)$. We provide only a sketch of the details, which requires the theory of prime ends (see, for example, [8, Section 17]).

Example 4.3. This example also uses $\mathbf{H}$ rather than $\mathbf{D}$. We define $g(z)=z / 2$, which is a holomorphic self-map of $\mathbf{H}$ with Denjoy-Wolff point 0 . Let $D$ be the simply connected domain shown in Figure 4.1. It is obtained by removing two vertical line 
segments and various horizontal line segments from $\mathbf{H}$ to leave an infinite snakelike domain, as suggested by the figure. There are infinitely many horizontal line segments, and they accumulate at the real interval $[-1,1]$, which is the impression of a unique prime end of $D$.

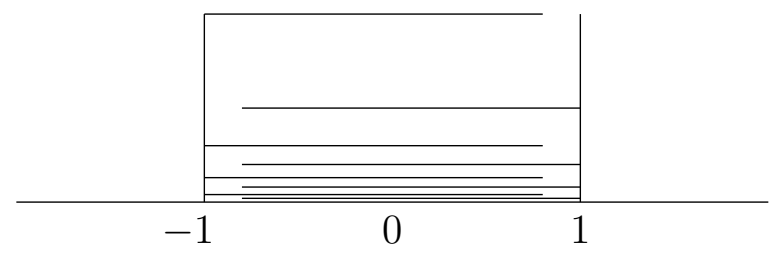

Figure 4.1. Domain D.

We define $h$ to be a conformal map from $\mathbf{H}$ to $D$. This map induces a one-to-one correspondence between the extended real line (the boundary of $\mathbf{H}$ ) and the prime ends of $D$. We choose $h$ such that 0 corresponds to the prime end with impression $[-1,1]$. Now consider the right-composition sequence $G_{n}=g_{1} g_{2} \cdots g_{n}$, where $g_{1}=h$ and $g_{n}=g$, for $n>1$. Then $\left(g_{n}\right)$ converges to $g$ as quickly as possible, however, we will show that $\left(G_{n}(i)\right)$ diverges. To see this, first observe that

$$
G_{n}(i)=h g^{n}(i)=h\left(i / 2^{n}\right), \quad \text { for } n=1,2, \ldots
$$

Since $h$ is a conformal map from $\mathbf{H}$ to $D$, it preserves hyperbolic distance between these two domains. So the hyperbolic length of the hyperbolic geodesic segment $\Gamma_{n}$ between $G_{n-1}(i)$ and $G_{n}(i)$ in $D$ is equal to the hyperbolic distance between $i / 2^{n-1}$ and $i / 2^{n}$ in $\mathbf{H}$, namely $\log 2$. Now, as $n$ increases, $i / 2^{n}$ approaches 0 (in the Euclidean metric), and $G_{n}(i)$ approaches $[-1,1]$ (in the Euclidean metric). By applying a simple estimate with the quasihyperbolic metric, it can then be shown that the Euclidean length of $\Gamma_{n}$ converges to 0 . From the shape of $D$ we can see that $\left(G_{n}(i)\right)$ accumulates at an interval within $[-1,1]$, so it diverges.

Example 4.3 indicates that there is little hope of obtaining a simple analogue of Theorem 4.2 for right-composition sequences. It also suggests that we ought to shift our perspective when considering right-composition sequences, in the following sense. The sequence $\left(G_{n}(i)\right)$ certainly diverges in the closure of the domain $\mathbf{H}$, but it converges in the Carathéodory compactification of the domain $D$, to the prime end with impression $[-1,1]$. In general, for a right-composition sequence $G_{n}=g_{1} g_{2} \cdots g_{n}$ acting on $\mathbf{D}$, it is likely to be more rewarding to consider convergence of $\left(G_{n}\right)$ not with respect to $\mathbf{D}$, but with respect to the set $\bigcap G_{n}(\mathbf{D})$ (or perhaps its interior), which in many cases will be a simply connected domain. We will examine this idea more thoroughly in future work.

\section{References}

[1] BAKer, I. N., and P. J. Rippon: Towers of exponents and other composite maps. - Complex Variables Theory Appl. 12:1-4, 1989.

[2] Beardon, A.F.: Continued fractions, discrete groups and complex dynamics. - Comput. Methods Funct. Theory 1:2, 2001, 535-594.

[3] Beardon, A. F.: Semi-groups of analytic maps. - Comput. Methods Funct. Theory, 1:1, 2001, 249-258.

[4] Christodoulou, A., and I. Short: A hyperbolic-distance inequality for holomorphic maps. - Ann. Acad. Sci. Fenn. Math. 44:1, 2019, 293-300. 
[5] GiLl, J.: Compositions of analytic functions of the form $F_{n}(z)=F_{n-1}\left(f_{n}(z)\right), f_{n}(z) \rightarrow f(z)$. - J. Comput. Appl. Math. 23:2, 1988, 179-184.

[6] Gill, J.: Complex dynamics of the limit periodic system $F_{n}(z)=F_{n-1}\left(f_{n}(z)\right), f_{n} \rightarrow f$. In: Extrapolation and rational approximation (Luminy, 1989), J. Comput. Appl. Math. 32:1-2, 1990, 89-96.

[7] Lorentzen, L., Compositions of contractions. - In: Extrapolation and rational approximation (Luminy, 1989), J. Comput. Appl. Math. 32:1-2, 1990, 169-178.

[8] Milnor, J.: Dynamics in one complex variable. Third edition. - Ann. of Math. Stud. 160, Princeton Univ. Press, Princeton, NJ, 2006.

[9] Pommerenke, Ch.: On asymptotic iteration of analytic functions in the disk. - Analysis 1:1, $1981,45-61$.

[10] Schiff, J.L.: Normal families. - Universitext, Springer-Verlag, New York, 1993.

Received 1 October $2019 \bullet$ Accepted 28 May 2020 